\title{
An All-Silicon Single-Wafer Micro-g Accelerometer with a Combined Surface and Bulk Micromachining Process
}

\author{
Navid Yazdi and Khalil Najafi, Fellow, IEEE
}

\begin{abstract}
This paper reports an all-silicon fully symmetrical $z$-axis micro-g accelerometer that is fabricated on a single-silicon wafer using a combined surface and bulk fabrication process. The microaccelerometer has high device sensitivity, low noise, and low/controllable damping that are the key factors for attaining $\mu \mathrm{g}$ and sub- $\mu \mathrm{g}$ resolution in capacitive accelerometers. The microfabrication process produces a large proof mass by using the whole wafer thicknes and a large sense capacitance by utilizing a thin sacrificial layer. The sense/feedback electrodes are formed by a deposited 2-3 $\mu \mathrm{m}$ polysilicon film with embedded $25-35 \mu \mathrm{m}$-thick vertical stiffeners. These electrodes, while thin, are made very stiff by the thick embedded stiffeners so that force rebalancing of the proof mass becomes possible. The polysilicon electrodes are patterned to create damping holes. The microaccelerometers are batch-fabricated, packaged, and tested successfully. A device with a $2-\mathrm{mm} \times 1-\mathrm{mm}$ proof mass and a full bridge support has a measured sensitivity of $2 \mathrm{pF} / \mathrm{g}$. The measured sensitivity of a 4-mm $\times 1-\mathrm{mm}$ accelerometer with a cantilever support is $\mathbf{1 9 . 4}$ $\mathrm{pF} / \mathrm{g}$. The calculated noise floor of these devices at atmosphere are $0.23 \mu \mathrm{g} / \sqrt{ } \mathrm{Hz}$ and $0.16 \mu \mathrm{g} / \sqrt{ } \mathrm{Hz}$, respectively.

[567]
\end{abstract}

\section{INTRODUCTION}

$\mathbf{H}$ IGH-precision microaccelerometers are increasingly needed in numerous applications including self-contained or GPS-augmented navigation and guidance systems, seismometry for oil-exploration and earthquake prediction, microgravity measurements and platform stabilization in space, and underwater acoustic measurements. While recent advances in fabrication technologies for microaccelerometers have made the mass production of these devices with low/medium sensitivities possible [1], [2], to date, only a few micro-g silicon accelerometers have been reported [3]-[8]. These devices utilize a large proof mass in conjunction with capacitive [3]-[6], resonant [7], or tunneling current [8] sensing schemes to achieve high sensitivity. Among all these, capacitive accelerometers have become more attractive and promising for high precision micro-g accelerometers due to their high sensitivity, good dc response and noise performance, low drift, low temperature sensitivity, low-power dissipation, large readout bandwidth, and simple structure.

Manuscript received April 18, 2000. This work was supported by the Defense Advanced Research Projects Agency under Contracts JFBI 92-149, DABT63-95- C-011, and F30602-98-2-0231. Subject Editor, N. deRooij.

N. Yazdi is with the Department of Electrical Engineering, Arizona State University, Tempe, AZ 85287-5706 USA (e-mail: yazdi@asu.edu).

K. Najafi is with the Center for Integrated Microsystems University of Michigan, Ann Arbor, MI 48109-2122 USA.

Publisher Item Identifier S 1057-7157(00)10871-6.
The large proof mass of the reported micro-g accelerometers is typically formed by wafer bonding and bulk micromachining [3]-[8] or, in some cases, by thick electroplated structures and the LIGA process [9]. Multiple wafer bonding is a viable threedimensional (3-D) fabrication technology for microsensors and microactuators [10]. However, silicon-silicon wafer bonded accelerometers require high temperature processing and could potentially drift. Post-bonding high-temperature treatment and anneal has been suggested to relieve the stresses and minimize drift in silicon-silicon bonded structures [11]. Glass and silicon wafers can be anodically bonded at lower temperature with a much simpler process. However, the difference between temperature coefficient of expansion of glass and silicon causes higher temperature sensitivity in the fabricated structures. In addition, the wafer bonding process results in thick structural layers that are desirable for forming the proof mass; nevertheless, reducing damping by incorporating damping holes in the thick bonded structural layers is difficult. LIGA devices are not typically inertial grade due to their relatively large temperature sensitivities and potentially long-term drift caused by creep and fatigue in metallic structures. Finally, surface micromachined accelerometers [12] have the main advantages of being monolithically fabricated with the interface circuit and increasing $Q$ by using damping holes. However, the proof mass in these devices is small, which results in lower sensitivity and typically above micro-g mechanical noise floor unless the accelerometer is vacuum packaged.

This paper presents an all-silicon fully symmetrical $z$-axis micro-g accelerometer that is fabricated on one silicon wafer using a combined surface and bulk fabrication process [13]. The microaccelerometer has high device sensitivity, low noise, and low/controllable damping, which are the key factors for attaining $\mu \mathrm{g}$ and sub- $\mu \mathrm{g}$ resolution in capacitive accelerometers. The high device performance is obtained by the novel device structure and fabrication process that enable formation of a large proof mass, controllable/small damping, and a narrow uniform air gap over a large area. In the following sections, the issues involved in the development of capacitive micro-g accelerometers are first reviewed. Then, the device structure, design, and fabrication process are discussed. Finally, fabrication and measurement results are presented.

\section{MiCRO-G ACCELEROMETER ISSUES}

A precision microaccelerometer is typically operated closedloop to obtain higher bandwidth, full-scale range, and linearity. 
Therefore, the main design consideration for these devices is meeting the resolution specification. Resolution of a microaccelerometer is determined by its mechanical noise and readout electronics noise. The electrical signal-to-noise ratio (SNR) can be improved by enhancing the sensor and the readout circuit sensitivity and reducing the circuit electrical noise. The mechanical noise is caused by the Brownian motion of the gas molecules surrounding the proof mass and the Brownian motion of the proof mass suspension or anchors. The total noise equivalent acceleration (TNEA $\left[\mathrm{m} / \mathrm{s}^{2} \sqrt{\mathrm{Hz}}\right]$ ) is shown to be [14]

$$
\mathrm{TNEA}=\frac{\sqrt{4 K_{B} T D}}{M}=\sqrt{\frac{4 K_{B} T \omega_{r}}{Q M}}
$$

where

$\begin{array}{ll}K_{B} & \text { Boltzmann constant; } \\ T & \text { temperature in Kelvin; } \\ D & \text { damping factor; } \\ M & \text { proof mass; } \\ \omega_{r} & \text { mechanical self-resonance frequency; } \\ Q & \text { quality factor. }\end{array}$

As is evident, mechanical noise can be reduced by increasing the proof mass size and reducing damping. Device damping is caused by both structural and viscous damping. However, the structural component is orders of magnitude smaller than the gas viscous damping even at low pressures (a few mTorr) and can be neglected [15]. Accelerometer damping can be decreased by using damping holes or vacuum packaging. Vacuum packaging of the accelerometer increases the sensor cost and manufacturing complexity. In addition, it may result in long-term drift due to package leakage. Using damping holes has none of these issues. Furthermore, it allows control of the damping factor by changing the hole density and geometry, which effectively simplifies the design of control circuitry for stable closed-loop operation. The damping factor of a perforated plate with equally spaced damping holes can be expressed by (2) [16]

$$
\begin{aligned}
D & =0.427 N \mu \frac{L^{4}}{d^{3}} \\
L & =\sqrt{p^{2}-a^{2}}
\end{aligned}
$$

where

$N \quad$ number of the damping holes;

$\mu \quad$ viscosity of the surrounding gas;

$d \quad$ air gap;

$L \quad$ effective plate length and is related to the damping hole size $(a)$ and pitch $(p)$ by (3).

In order to demonstrate the effectiveness of damping holes in reducing the damping factor and lowering the mechanical noise floor, an accelerometer TNEA versus the proof mass thickness at atmospheric pressure for three different cases-a) 4- $\mu \mathrm{m}$ damping holes with $9-\mu \mathrm{m}$ pitch; b) 5- $\mu \mathrm{m}$ damping holes with $15-\mu \mathrm{m}$ pitch; c) without any damping holes-is plotted in Fig. 1. The device has a mass/electrode area of $2 \mathrm{~mm} \times 1$ $\mathrm{mm}$, is at atmospheric pressure, and has a $1.5 \mu \mathrm{m}$ capacitive air gap between the electrode and the proof mass. Squeeze film damping and mechanical noise are obtained using (1) and (2).

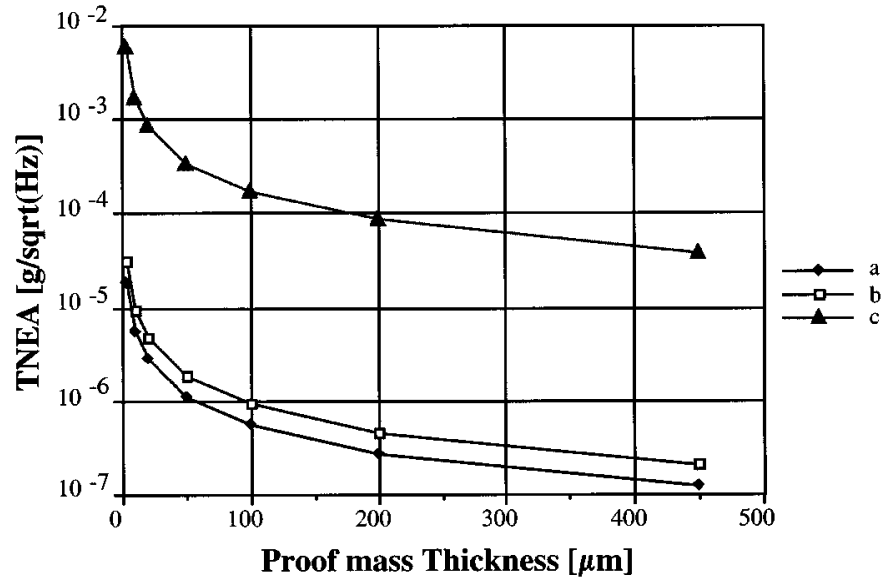

Fig. 1. Total noise equivalent acceleration (TNEA) due to Brownian motion at atmospheric pressure for an accelerometer with $1 \mathrm{~mm} \times 2 \mathrm{~mm}$ mass/electrode area and $1.5 \mu \mathrm{m}$ air gap with (a) $4-\mu \mathrm{m}$ damping holes with $9-\mu \mathrm{m}$ pitch, (b) $5-\mu \mathrm{m}$ damping holes with $15-\mu \mathrm{m}$ pitch, and (c) no damping holes.

As can be observed, a sub-micro-g noise floor is achievable at atmosphere by using both thick proof mass and damping holes. Note that by forming the damping holes in the electrode rather than the proof mass, there is no loss in the mass size. Furthermore, by using small damping holes, the fringing capacitance becomes comparable with the lost capacitance due to reduction in surface area caused by the formation of damping holes, and thus, there is almost no loss of sense capacitance.

The overall sensor SNR can be also improved by increasing the open-loop sensitivity and enhancing the sense signal at the input of the readout circuit. The static open-loop sensitivity of the accelerometer with capacitive area of $A$ and air gap of $d$ can be expressed by

$$
\frac{\Delta C_{\text {static }}}{a}=\frac{\varepsilon_{0} A M}{d^{2} K}=\frac{\varepsilon_{0} A}{d^{2} \omega_{r}^{2}} .
$$

Thus, open-loop sensitivity improves linearly with increasing proof mass thickness and electrode area. It is also inversely proportional to the air gap squared and the suspension spring constant. In summary, the challenge in developing a capacitive micro-g accelerometer is to attain thick large mass, soft suspension, narrow air gap over a large area, and high-quality factor simultaneously.

\section{Device Structure}

The microaccelerometer utilizes a novel device structure and fabrication process to address all the micro-g accelerometer design issues. The central idea in this structure is combining surface and bulk micromachining to fabricate devices with high sensitivity, low noise floor, and controllable damping on a single silicon wafer. Fig. 2 shows the accelerometer structure and its top and cross-sectional views. The main features of the device structure and its fabrication process are 1) use of the entire wafer thickness to attain a large proof mass, 2) use of a sacrificial thin film to form a uniform and conformal gap over a large area, and 3 ) use of deposited polysilicon and deep trench etching to create mechanically stiff electrodes on both sides of the proof mass circumventing the need for multiple wafer bonding. 


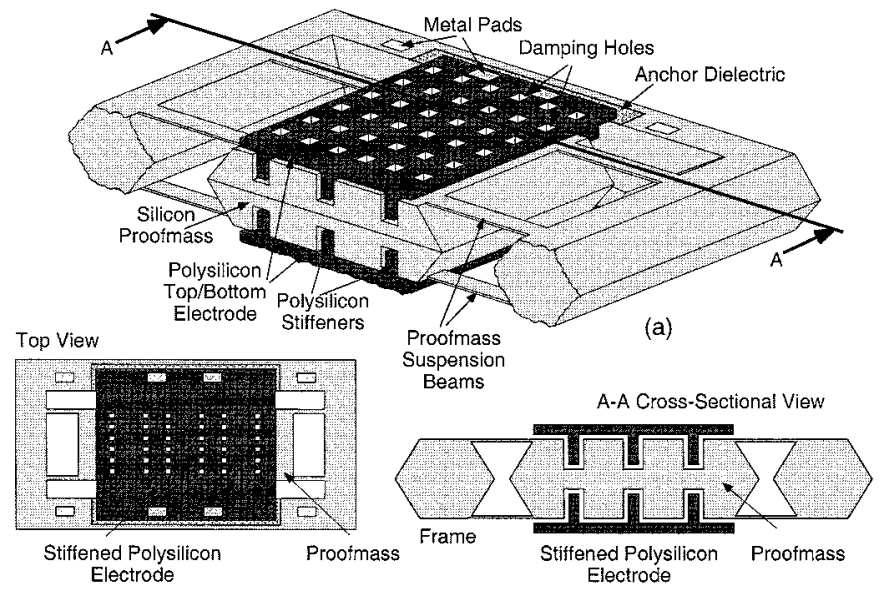

(c)

(b)

Fig. 2. Micro-g accelerometer structure. (a) Device structure. (b) A-A cross-sectional view. (c) Top view.

The electrodes, while thin, are made stiff in the sense direction by embedding thick vertical stiffeners in them. Thus these electrodes can be used to electrostatically force-rebalance the proof mass and provide closed-loop operation. The thick vertical stiffeners are formed by refilling high aspect-ratio trenches with thin polysilicon film deposition. Any damping hole configuration and geometry can be easily formed on the thin polysilicon electrodes to optimize damping factor and capacitance, while there is no concern with large hole density and its effect on the electrode softening as the plate stiffness is mainly provided by the embedded stiffeners.

The proof mass and its supporting rim have the whole wafer thickness and are formed by anisotropic etching of the silicon wafer (bulk micromachining). When the proof mass and the electrodes touch, electrical isolation is provided by dielectric layer stand-offs placed on the proof mass. There are eight suspension beams, which are symmetric with respect to the proof mass centerline and result in low cross-axis sensitivity. These suspension beams are formed using heavily boron-doped strips of the silicon wafer or deposited polysilicon layer. The sense and feedback electrodes are polysilicon plates created on both sides of the proof mass and anchored on an isolation dielectric at the frame. The device has low temperature sensitivity as the polysilicon electrodes and silicon frame thermal expansion coefficients match each other closely. Furthermore, the device has a good long-term stability as it is all-silicon and no wafer bonding is used in its fabrication process.

\section{DEVICE DESIGN}

\section{A. Proof Mass}

In order to increase the sensitivity and reduce the noise floor, the proof mass is desired to be as large as possible. Two different designs with proof mass of $4 \mathrm{~mm} \times 1 \mathrm{~mm}$ and $2 \mathrm{~mm} \times 1 \mathrm{~mm}$ with a thickness of $\approx 450 \mu \mathrm{m}$ for a 4 -in Si wafer were selected. Hence, the proof mass sizes are $2.1 \mathrm{mg}$ and $4.2 \mathrm{mg}$, respectively. The proof mass has a rectangular geometry to both keep the electrode lengths limited and obtain a large mass. The proof mass is formed by wet anisotropic silicon etching of (100) silicon wafer, and corner compensation patterns are used to avoid mask undercutting at the corners [17], [18].

\section{B. Suspension Beams}

The accelerometer structure is entirely symmetrical around its proof mass center point, and therefore, a low cross-axis sensitivity can be achieved for a cantilever support as well. The design uses a full-bridge support configuration with eight support $700 \mu \mathrm{m} \times 40 \mu \mathrm{m} \times 3 \mu \mathrm{m}$ heavily boron-doped Si beams that yield a spring constant of $65 \mathrm{~N} / \mathrm{m}$ with an intrinsic stress of $40 \mathrm{MPa}$. The beams are slightly misaligned relative to the wafer flat to allow their undercutting. A more compliant cantilever support can be obtained by cutting all the beams on one side of the proof mass.

\section{Air Gap}

The accelerometer sensitivity inversely increases with the air gap squared. The lower limit of the air gap is determined by the device release time and stiction. The air gap is defined by a sacrificial silicon oxide $\left(\mathrm{SiO}_{2}\right)$ layer and is wet etched in the final stage of device fabrication. This sacrificial layer is to be etched at the surface and deep in the proof mass trenches to separate the proof mass from the polysilicon electrodes and their vertical stiffeners. The etch front proceeds in the narrow long channels created by the sacrificial layer etch in the trenches and is a nonlinear function of the channel length and height [19]. With a $35-\mu \mathrm{m}$-deep trench, a conservative air gap size of $1.4 \mu \mathrm{m}$ provides a sufficiently short release time so that the metal and dielectric silicon nitride layers at the silicon frame are not significantly etched. In addition, a gap size of $1.4 \mu \mathrm{m}$ is adequately large to avoid severe yield loss due to stiction.

\section{Damping and Noise}

The damping factor is to be reduced sufficiently to lower the mechanical noise floor. In order to achieve a few micro-g resolution in a 100-Hz bandwidth and satisfy the requirements of the majority of the applications, a mechanical noise of a few tenth's of $\mu \mathrm{g} / \sqrt{ } \mathrm{Hz}$ is needed. The noise and the damping factor are controlled and reduced by forming damping holes in the electrodes [16]. It can be shown that for a square hole smaller than $18 \mu \mathrm{m}$ and an air gap of $1.4 \mu \mathrm{m}$, the fringing capacitance is, in fact, larger than the lost area capacitance. In addition, in order to avoid plate softening of the electrodes between the stiffeners, a low hole density of $<15 \%$-area is selected in the design. Considering all these design constraints, 5- $\mu \mathrm{m}$ damping holes with $15-\mu \mathrm{m}$ pitch are incorporated in the electrode plates. The damping factor of the accelerometer with $2 \mathrm{~mm} \times 1 \mathrm{~mm}$ proof mass is calculated to be 7 e- 4 N.m/s, which results in an equivalent noise floor of 0.23 $\mu \mathrm{g} / \sqrt{ } \mathrm{Hz}$ at atmosphere for the double-side accelerometer. The fringing filed capacitance for each hole is $0.43 \mathrm{fF}$, whereas the lost area capacitance is $0.16 \mathrm{fF}$.

\section{E. Sense and Feedback Electrodes}

The top and bottom electrodes are divided into 5 and 12 electrically isolated electrodes for $2 \mathrm{~mm} \times 1 \mathrm{~mm}$ and $4 \mathrm{~mm} \times 1$ $\mathrm{mm}$ devices, respectively. These electrodes each can be separately used for sense, feedback, self-test, or calibration of the accelerometer. However, the maximum sensitivity and dynamic range are obtained when all the electrodes are used for sense and feedback. 


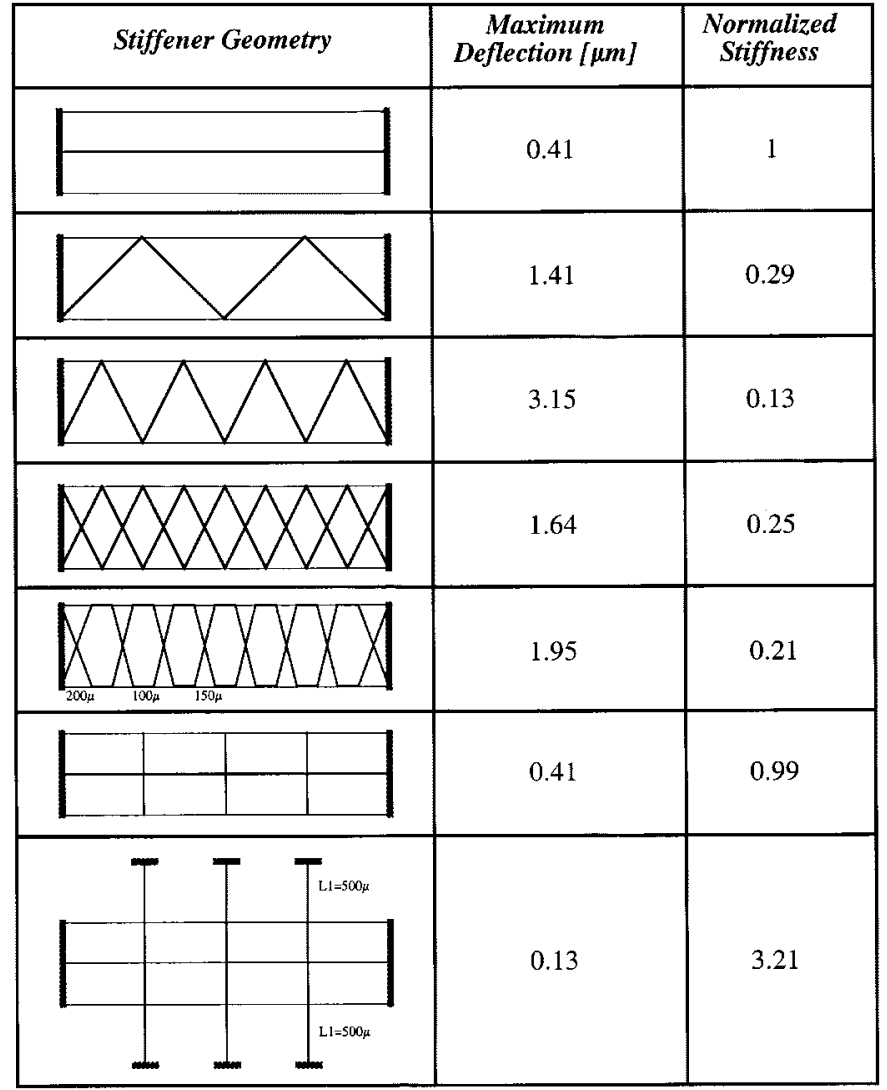

Fig. 3. Summary of comparative FEM simulations of electrodes with various stiffener geometeries. The electrode is $2000 \mu \mathrm{m} \times 200 \mu \mathrm{m} \times 2 \mu \mathrm{m}$, the stiffener height is $15 \mu \mathrm{m}$, the stiffener thickness is $2 \mu \mathrm{m}$, and the pressure load is $20 \mathrm{~Pa}$.

The sidewall capacitance of the stiffeners and proof mass contributes to the electrode parasitic capacitance. Thus, the stiffener design objective is to achieve sufficient rigidity with the minimum sidewall capacitance. In order to determine the most suitable stiffener geometry, stiffness of the electrode in the $z$ direction with a uniformly distributed pressure load was simulated for a number of different stiffener shapes. The comparative results of these FEM simulations are shown in Fig. 3. As can be observed, the stiffness of the plate with straight stiffener is the highest, and its sidewall area is the lowest. Hence, it is the most suitable choice. In the actual design, each electrode contains two vertical stiffeners $3.5 \mu \mathrm{m}$ wide and $30 \mu \mathrm{m}$ high separated from each other by $210 \mu \mathrm{m}$, and three perpendicular stiffeners are added to connect these main stiffeners together. The calculated stiffness of each electrode in this case assuming a negligible intrinsic stress for the $3-\mu \mathrm{m}$ polysilicon electrode is $\approx 510 \mathrm{~N} / \mathrm{m}$. This stiffness yields a maximum deflection of $16.1 \mathrm{~nm}$ under 29 Pa load ( $2 \mathrm{~g}$ acceleration with $2 \mathrm{~mm} \times 1 \mathrm{~mm}$ proof mass).

\section{F. Dimples}

The polysilicon electrodes contain dimples underneath to reduce the contact area between the proof mass and the electrode and, thus, help with alleviating stiction [20]. These dimples are aligned to the silicon nitride dielectric standoffs on the proof mass and provide electrical isolation as well. The dimple size is set by the fabrication process and is $3 \mu \mathrm{m}$ on a side rounded by wet etching. The dimple height limits the accelerometer open-loop dynamic range, and a high density of dimples increase the surface adhesion forces when the electrode and the proof mass touch. The design uses $0.4-\mu \mathrm{m}$ dimples separated 63 $\mu \mathrm{m}$ from each other.

\section{FABRICATION PROCESS}

The fabrication process requires eight masks and is performed symmetrically on both sides of the wafer, as shown in Fig. 4. The process starts with a shallow $(3 \mu \mathrm{m}) \mathrm{p}++$ boron diffusion on $\langle 100\rangle$ double-polished p-type $\mathrm{Si}$ wafer using thermal oxide as a mask. Both sides of the wafer are patterned, and the patterns are aligned to each other. The shallow boron diffusion is performed at $1150^{\circ} \mathrm{C}$ for $30 \mathrm{~min}$ and defines the beams, the proof mass, and the supporting rim. Then, 2200 $\AA$ of LPCVD nitride is deposited and patterned to form the polysilicon electrode anchors and isolation dielectric under the polysilicon electrode dimples. The next masking step is etching 30- $\mu \mathrm{m}$-deep, $6-\mu \mathrm{m}$-wide trenches to define the vertical electrode stiffeners.

The trenches are then refilled completely using $1.4-\mu \mathrm{m}$ sacrificial LPCVD oxide and 1.8- $\mu \mathrm{m}$ LPCVD polysilicon. The polysilicon is etched back using a blanket RIE etch, exposing the sacrificial oxide and leaving polysilicon plugs in the trenches. In this manner, the step height due to the trench etch is reduced, and sacrificial oxide at the bottom of the trenches is protected. Next, two patterning steps are performed on the sacrificial oxide. First, it is patterned and partially wet etched to form dimples inside the polysilicon electrodes. These dimples reduce the contact area and help to reduce stiction. Second, narrow strips of sacrificial oxide are patterned and etched away at the anchors. Sacrificial oxide is left in most areas of the anchors to reduce parasitics and is sealed between underneath nitride and top polysilicon. A $2-3-\mu \mathrm{m}$ LPCVD polysilicon is then deposited to connect to the plugs in the trenches and form the electrodes. The polysilicon electrode is doped with phosphorus or boron halfway through deposition. The electrodes are sealed with a 4000- $\AA$ LPCVD oxide in the next step. This oxide is patterned to form metal contacts and openings to bulk $\mathrm{Si}$ for the subsequent proof mass release. The wafer is then patterned for $\mathrm{Cr} / \mathrm{Au}(400-\AA / 5000-\AA)$ sputtering and liftoff. The proof mass is released by an EDP etch at $110^{\circ} \mathrm{C}$ and following sacrificial oxide etch in 1:1 HF : DI-water. Then, the released structures are dried in an oven after a methanol soak or coated with self-assembled monolayer (SAM). SAM coating makes the polysilicon and silicon surfaces hydrophobic and helps substantially with preventing stiction [21].

\section{FABRICATION AND MEASUREMENT RESUlTS}

A number of prototype microaccelerometers have been successfully fabricated. Fig. 5 shows a SEM view of the device with $2 \mathrm{~mm} \times 1 \mathrm{~mm}$ proof mass. A rectangular proof mass has been utilized for this device to achieve a large mass without making the electrodes too long. The device has five electrically isolated electrodes on each side, which are anchored at the rim using stiffened polysilicon supports. An enlarged view of a single electrode stiffener support is presented in Fig. 6. The electrode 


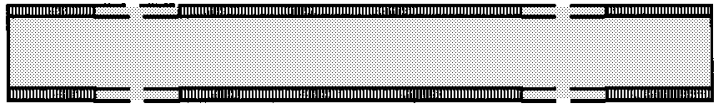

(a) Shallow boron diffusion to define beams, proofmass, and supporting rim. Nitride depositon and definiton for electrode anchors and isolation dielectric underneath electrode dimples.

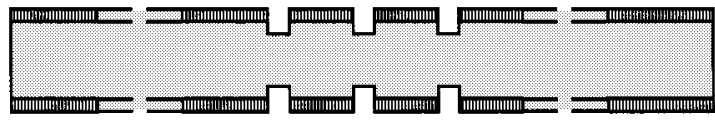

(b) Trench etch for vertical electrode stiffener definition.

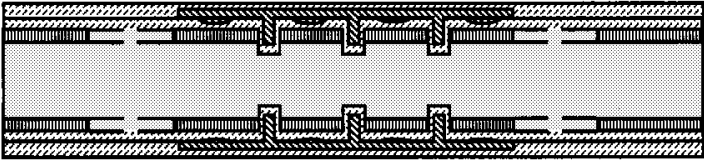

(c) Trench refill using LPCVD sacrifical oxide and poly. Poly patterning to form electrodes and damping holes. Poly is sealed by a top LPCVD oxide layer. Dimples inside poly elecrode are created by partial etching of the sacrifical oxide before poly deposition.

Fig. 4. Fabrication process sequence of the symmetrical $\mu \mathrm{g}$ accelerometer.

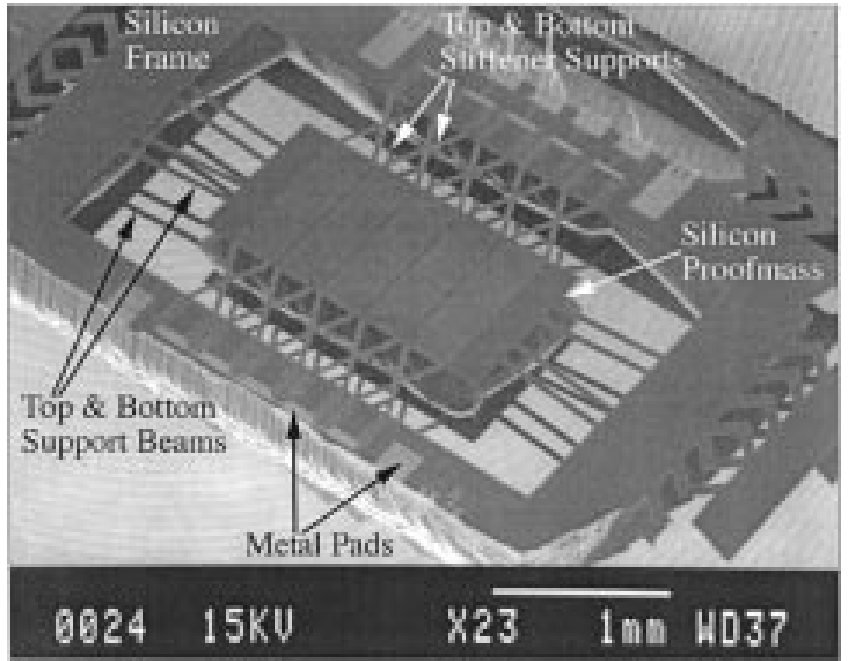

Fig. 5. SEM view of a high-precision symmetric $\mu \mathrm{g}$ accelerometer with $2 \mathrm{~mm}$ $\times 1 \mathrm{~mm}$ proof mass.

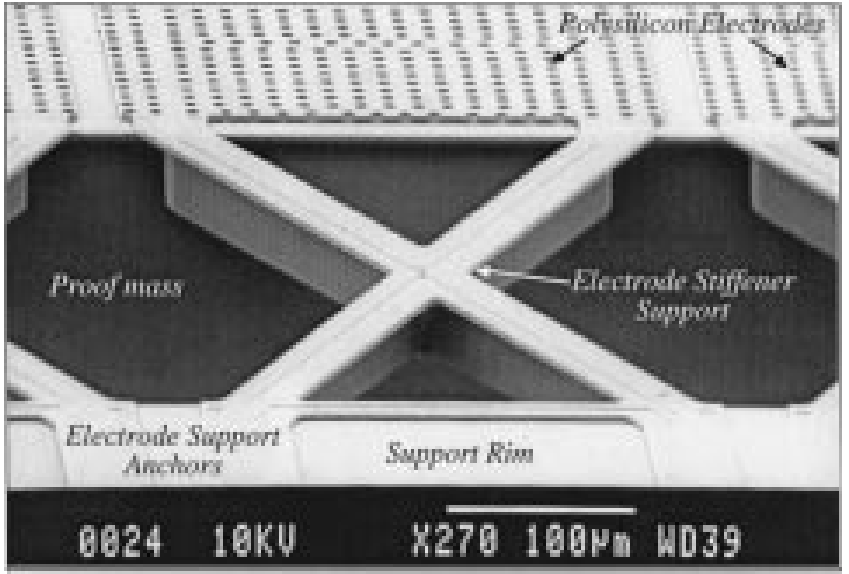

Fig. 6. Enlarged view of single electrode stiffener support. The electrode damping holes can be seen as well.

damping holes can be also seen in this figure. Fig. 7 shows a close-up view of the polysilicon electrode, its vertical embedded

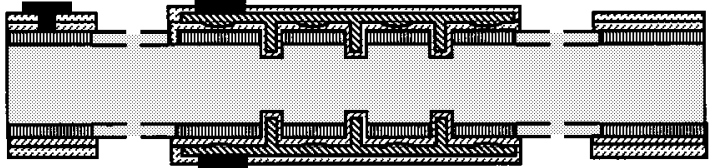

(d) Contact opening, metal deposition and patterning

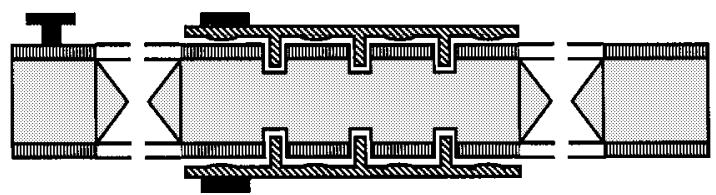

(e) Proofmass and rim formation by etching in EDP.

Final proofmass release by etching the sacrifical oxide.
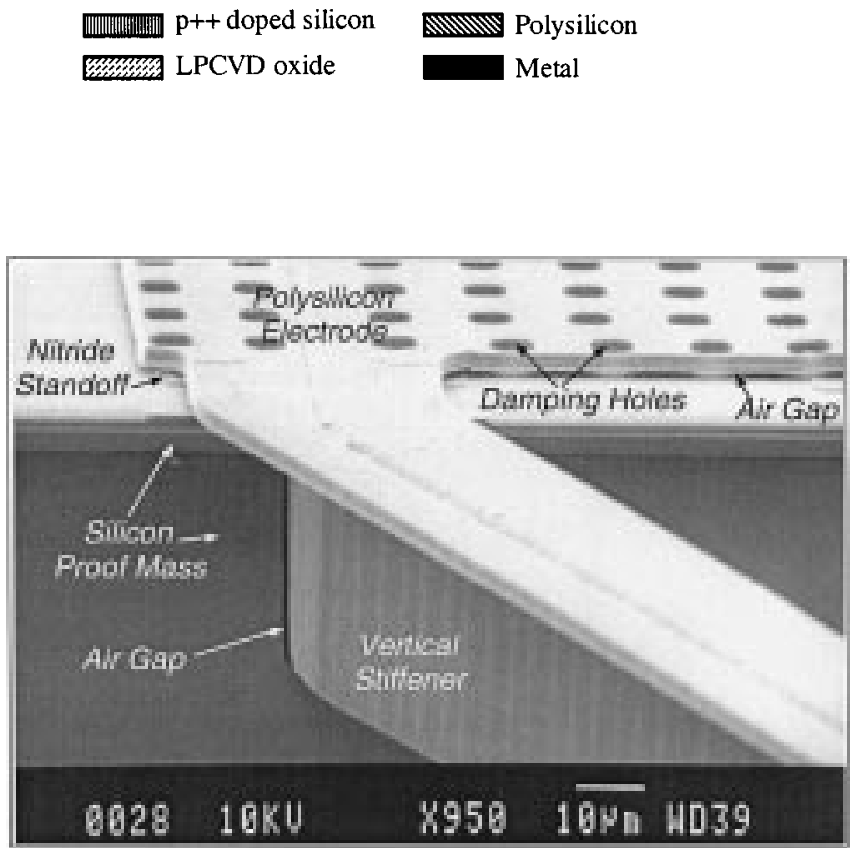

Fig. 7. Close-up SEM view of polysilicon electrode, its vertical embedded stiffener, and the proof mass. Electrode damping holes and isolating nitride standoffs can be seen as well.

stiffener, and the silicon proof mass. The isolating nitride standoffs can be seen in this SEM as well. A close-up view of the cross-section of the proof mass and stiffened polysilicon electrode is shown in Fig. 8. As is observed, the air gap conformally separates the electrode stiffener from the proof mass.

The fabricated accelerometers are tested electrostatically and mechanically on a turntable. Fig. 9 shows the measured open-loop response of a microaccelerometer with $2 \mathrm{~mm} \times 1$ $\mathrm{mm}$ proof mass with a full-bridge support. Electrostatic force is used to generate input acceleration in this measurement. Small displacement of the electrodes is taken into account by measuring spring constant of the electrode independently using the included test structures. The device shows a sensitivity of 2 $\mathrm{pF} / \mathrm{g}$, and the calculated sensitivity is $2.2 \mathrm{pF} / \mathrm{g}$, as presented in Table I. The smaller measured sensitivity is possibly due to the slightly thicker suspension beams $(>3 \mu \mathrm{m})$ and the smaller proof mass. The sensitivity can be increased by cutting half of the beams on one side and forming a cantilever support. The turntable test has shown a sensitivity of $19.4 \mathrm{pF} / \mathrm{g}$ on each side of the 4-mm $\times 1-\mathrm{mm}$ accelerometer with a cantilever support. 


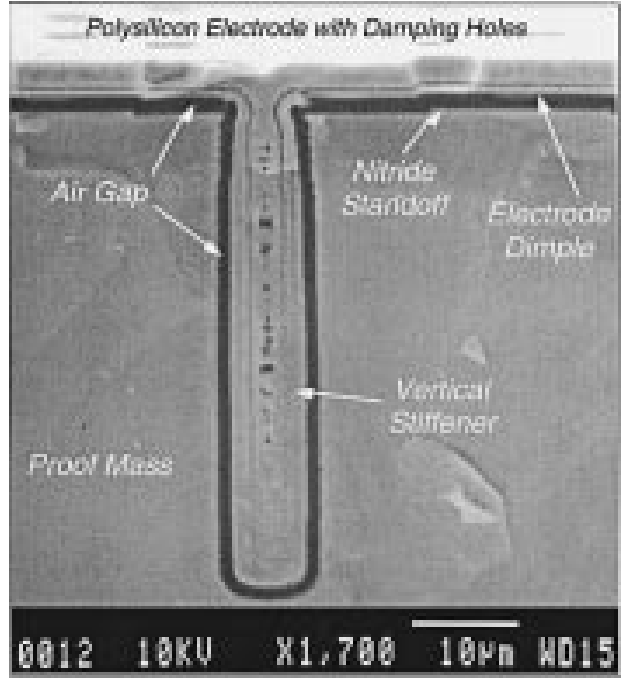

Fig. 8. Cross-sectional close-up of the proof mass and the stiffened polysilicon electrode.

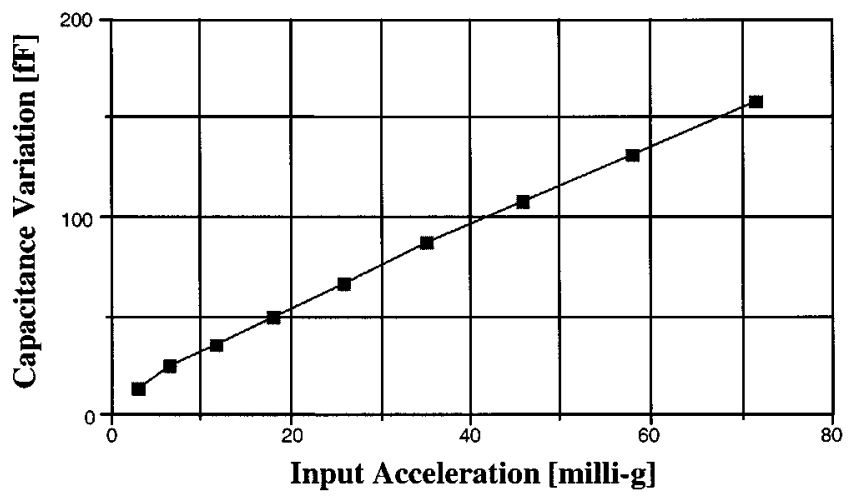

Fig. 9. Measured open-loop response of microaccelerometer with 2-mm $\times$ 1-mm proof mass and full-bridge support.

TABLE I

SUMMARY OF THE DESIGN AND MEASUREMENT PARAMETERS FOR THE 2-mm $\times 1-$ mm ACCELEROMETER WITH A FULL-BRIDGE SUPPORT

\begin{tabular}{c|c|c}
\hline Parameter & Calculated & Measured \\
\hline Proof Mass Size & 2.1 milligram & - \\
\hline Suspension Beam Stiffness & $65 \mathrm{~N} / \mathrm{m}$ & - \\
\hline Resonant Frequency & $885 \mathrm{~Hz}$ & - \\
\hline Air Gap & - & $1.4 \mu \mathrm{m}$ \\
\hline Number of Electrodes & 5 & 5 \\
\hline Electrode Size & - & $210 \mu \mathrm{m} \times 2000 \mu \mathrm{m} \times 3 \mu \mathrm{m}$ \\
\hline Electrode Stiffener & - & $3.5 \mu \mathrm{m} \times 2000 \mu \mathrm{m} \times 30 \mu \mathrm{m}$ \\
(2 per electrode) & & $1135 \mathrm{~N} / \mathrm{m}$ \\
\hline Electrode Stiffness & $510 \mathrm{~N} / \mathrm{m}$ & $67.5 \mathrm{pF}$ \\
\hline Base Capacitance & $76 \mathrm{pF}$ & - \\
\hline Sense Capacitance & $6 \mathrm{pF}$ & - \\
\hline Parasitic Capacitance & $70 \mathrm{pF}$ & $2 \mathrm{pF} / \mathrm{g}$ \\
\hline Open-Loop Sensitivity & $2.2 \mathrm{pF} / \mathrm{g}$ & - \\
\hline Quality Factor (Q) & 16.7 & - \\
\hline Mechanical Noise @ Atmosphere & $<0.23 \mu \mathrm{g} / \mathrm{vHz}$ &
\end{tabular}

In order to measure the stiffness of the electrodes, the bulk silicon was partially etched out to form the post-release device sense capacitors without releasing the proof mass. The sacrificial oxide layer was etched entirely to release the electrodes. These electrodes were used to measure the pull-in voltage and obtain their stiffness. As the dc sweep bias is increased, two pull-in voltages are observed: i) The first pull-in occurs with $19.3-\mathrm{V}$ bias that corresponds to the electrode collapsing on the proof mass

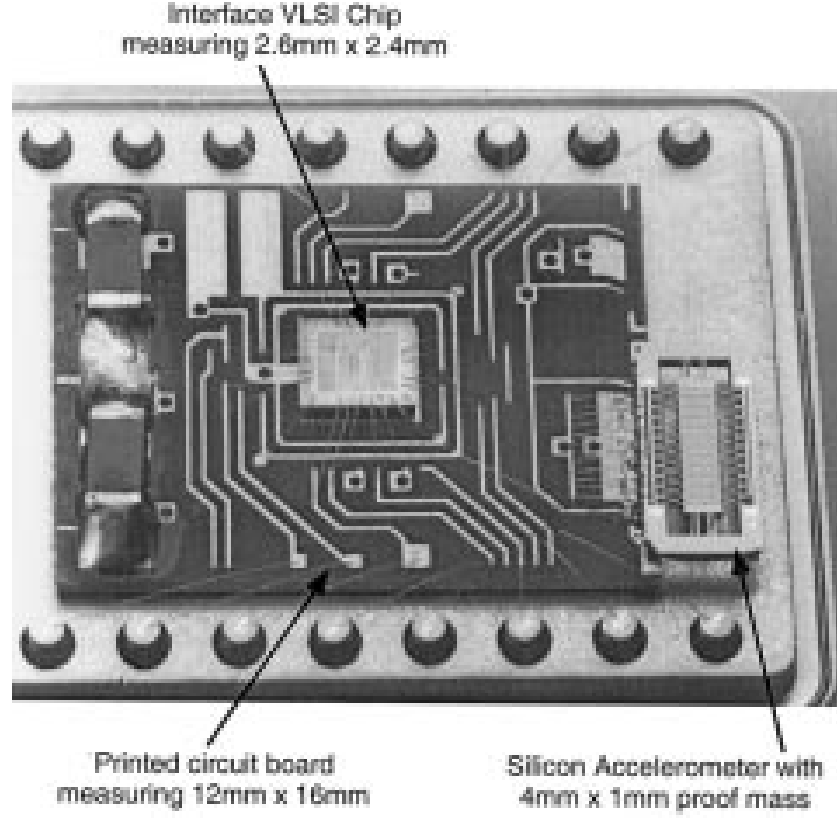

Fig. 10. Hybrid packaged accelerometer and the interface chip in a standard 24-pin IC package. Accelerometer is mounted a single edge to reduce the packaging-induced stresses.

and resting on its dimples. The plate stiffness corresponding to the measured pull-in voltage is $1135 \mathrm{~N} / \mathrm{m}$. This measured value is higher than $510 \mathrm{~N} / \mathrm{m}$ calculated stiffness (see Section IV) that is due to a tensile stress of the boron doped polysilicon plate. ii) The second pull-in happens at $27.8 \mathrm{~V}$, which is caused by the electrode collapsing on the proof mass in the center between four adjacent dimples. The associated stiffness of the plate segment between the four dimples is $1452 \mathrm{~N} / \mathrm{m}$. A summary of the design and measured parameters for the $2-\mathrm{mm} \times 1-\mathrm{mm}$ accelerometer with a full-bridge support is presented in Table I.

\section{PACKAGING}

After completion of the fabrication, the microaccelerometer is mounted on one edge of its frame on a recessed substrate and is suspended over the recessed area. In this manner, the package and mounting-induced stresses will be reduced but not affect the device performance. The device has bonding pads on both sides. Each pad at the bottom has a metal overhang over the frame, which is directly bonded to the pads on the mounting substrate using ultrasonic wirebonder and provide beam-lead transfer [22]. Access to the top pads is provided by direct wirebonding to them. Fig. 10 shows the hybrid packaged accelerometer, its interface circuit [23], and the reference capacitors in a standard DIP IC package. This package will be ideally sealed to protect the sensor and the circuit from environmental humidity, which may affect the parasitics or potentially cause the sensor failure due to stiction.

\section{CONCLUSION}

In this paper, an all-silicon single-wafer fully symmetrical capacitive micro-g accelerometer is presented. This microaccelerometer has high device sensitivity, low noise, low/controllable damping, low cross-axis sensitivity, low temperature sensi- 
tivity, and potentially good long-term stability. All these features are achieved by a novel device structure and a new combined surface and bulk microfabrication process that overcome the micro-g accelerometer design challenges. Two different micro-g accelerometer designs with proof mass sizes of $2 \mathrm{~mm} \times 1 \mathrm{~mm}$ and $4 \mathrm{~mm} \times 1 \mathrm{~mm}$ are successfully batch fabricated, packaged, and tested. The device with $2-\mathrm{mm} \times 1$-mm proof mass and a full-bridge support has a measured sensitivity of $2 \mathrm{pF} / \mathrm{g}$. The measured sensitivity of the 4-mm $\times 1-\mathrm{mm}$ accelerometer with a cantilever support is $19.4 \mathrm{pF} / \mathrm{g}$, which yields a differential top and bottom sensitivity of $38.8 \mathrm{pF} / \mathrm{g}$. The calculated noise floor of these devices at atmosphere are $0.23 \mu \mathrm{g} / \sqrt{ } \mathrm{Hz}$ and $0.16 \mu \mathrm{g} / \sqrt{ } \mathrm{Hz}$, respectively.

\section{REFERENCES}

[1] “ADXL50-monolithic accelerometer with signal conditioning," Analog Devices, Norwood, MA, 1993.

[2] "MMAS40G10D-Micromachined accelerometer," Motorola, Phoenix, AZ, 1997.

[3] F. Rudolf, A. Jornod, J. Berqvist, and H. Leuthold, "Precision accelerometers with $\mu \mathrm{g}$ resolution," Sensors Actuators, vol. A21-A23, pp. 297-302, 1990.

[4] W. Henrion, L. DiSanza, M. Ip, S. Terry, and H. Jerman, "Wide-dynamic range direct digital accelerometer," in Tech. Dig. Solid-State Sensors Actuators Workshop, Hilton Head Island, SC, June 1990, pp. 153-156.

[5] K. Warren, "Navigation grade silicon accelerometer with sacrifically etched SIMOX and BESOI structure," in Tech. Dig. Solid-State Sensors Actuators Workshop, Hilton Head Island, SC, June 1994, pp. 69-72.

[6] J. Bernstein, R. Miller, W. Kelley, and P. Ward, "Low-noise MEMS vibration sensor for geophysical applications," J. Microelectromech. Syst., vol. 8, pp. 433-438, Dec. 1999

[7] T. V. Roszhart, H. Jerman, J. Drake, and C. de Cotiis, "An inertial-grade micromachined vibrating beam accelerometer," in Tech. Dig. Eighth Int. Conf. Solid-State Sensors Actuators, Stockholm, Sweden, June 1995, pp. 656-658.

[8] C. Liu, A. M. Brazilai, J. K. Reynolds, A. Partridge, T. W. Kenny, J. D. Grade, and K. Rockstad, "Characterization of a high-sensitivity micromachined tunneling accelerometer with micro-g resolution," J. Microelectromech. Syst., vol. 2, pp. 235-244, June 1998.

[9] O. Kromer, O. Fromhein, H. Gemmeke, T. Kuhner, J. Mohr, and M. Strohramann, "High-precision readout circuit for LIGA acceleration sensors," Sensors Actuators, vol. 46-47, pp. 196-200, 1995.

[10] M. A. Schmidt, "Wafer-to-wafer bonding for microstructure formation," Proc. IEEE, vol. 86, pp. 1575-1585, Aug. 1998.

[11] M. L. Kniffin and M. Shah, "Packaging for silicon micromachined accelerometers," Int. J. Microcircuits Electron. Packag., vol. 19, no. 1, pp. 75-86, First quarter 1996.

[12] B. Boser and R. T. Howe, "Surface micromachined accelerometers," IEEE J. Solid-State Circuits, vol. 31, pp. 366-375, Mar. 1996.

[13] N. Yazdi and K. Najafi, "An all-silicon single-wafer fabrication technology for precision microaccelerometers," in Tech. Dig. Ninth Int. Conf. Solid-State Sensors Actuators , Chicago, IL, June 1997, pp. 1181-1184.

[14] T. B. Gabrielson, "Mechanical-thermal noise in micromachined acoustic and vibration sensors," IEEE Trans. Electron Devices, vol. 40, pp. 903-909, May 1993.

[15] J. D. Zook et al., "Characteristics of polysilicon resonant microbeams," Sensors Actuators, pp. 51-59, Oct. 1992

[16] V. M. McNeil, M. Novack, and M. A. Schmidt, "Design and fabrication of thin-film microaccelerometer using wafer bonding," in Proc. IEEE Int. Conf. Sensors Actuators, Yokohama, Japan, June 1993, pp. 822-825.

[17] B. Puers and W. Sansen, "Compensation structures for convex corner micromachining in silicon," Sensors Actuators, vol. A21-A23, pp. 1036-1041, 1990.

[18] C. Standman, L. Rosengern, H. Elderstig, and Y. Bucklund, "Fabrication of $45^{\circ}$ mirrors together with well-defined V-grooves using wet anisotropic etching of silicon," J. Microelectromech. Syst., vol. 4, pp. 213-219, Dec. 1995.

[19] D. A. Monk, D. S. Soane, and R. T. Howe, "Sacrificial layer $\mathrm{SiO} 2$ wet etching for micromachining applications," in Tech. Dig. Sixth Int. Conf. Solid-State Sensors Actuators, San Francisco, CA, June 1991, pp. 647-650.
[20] C. H. Mastragelo and C. H. Hsu, "Mechanical stability and adhesion of microstructures under capillary forces-Part II: Experiments," J. Microelectromech.Syst., vol. 2, pp. 44-55, Mar. 1993.

[21] U. Srinivasan, M. R. Houston, R. T. Howe, and R. Maboudian, "Self-assembeled fluorcarbon films for enhanced stiction reduction," Tech. Dig. Int. Conf. Solid-State Sensors Actuators, pp. 1399-1402, June 1997.

[22] Q. Bai and K. D. Wise, "A high-yield process for three-dimensional microelectrode arrays," in Tech. Dig. Solid-State Sensors Actuators Workshop, Hilton Head Island, SC, June 1996, pp. 262-265.

[23] N. Yazdi and K. Najafi, "An interface IC for a capacitive silicon $\mu \mathrm{g}$ accelerometer," in Tech. Dig. IEEE Int. Solid-State Circuits Conf., San Francisco, CA, Feb. 1999, pp. 132-133.

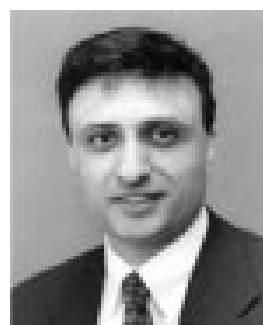

Navid Yazdi received the B.S. degree (with honors) in 1988 from the University of Tehran, Tehran, Iran, the M.S. degree in 1993 from the University of Windsor, Windsor, ON, Canada, and the Ph.D. degree in 1999 from the University of Michigan, Ann Arbor, all in electrical engineering.

Since November 1998, he has been an Assistant Professor with the Department of Electrical Engineering, Arizona State University, Tempe. From 1988 to 1991 , he was an R\&D Engineer at University of Tehran, where he worked on hardware/software development for Computer Numerical Control (CNC) machine tools and large data acquisition systems. From 1991 to 1993, he worked on architectures and mixed-signal circuit design for VLSI implementation of artificial neural networks at the University of Windsor. Since 1993, his research work at the University of Michigan has been focused on high-performance mixed-signal interface circuits for microsensors, high-precision microaccelerometer design and fabrication, electromechanical oversampled sigma-delta modulators, and wireless telemetry for microsystems. His research interests include integrated sensors and actuators, micromachining and microstructure fabrication technologies, and mixed analog-digital interface circuits for microelectromechanical systems.

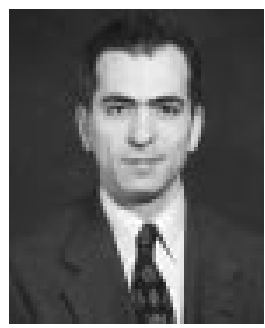

Khalil Najafi (S'84-M'86-SM'97-F'00) was born in 1958. He received the B.S., M.S., and the Ph.D. degrees in 1980, 1981, and 1986, respectively, all in electrical engineering from the Department of Electrical Engineering and Computer Science, University of Michigan, Ann Arbor.

From 1986 to 1988, he was a Research Fellow, from 1988 to 1990 an Assistant Research Scientist, from 1990 to 1993 an Assistant Professor, from 1993 to 1998 an Associate Professor, and since September 1998 a Professor and the Director of the Solid-State Electronics Laboratory, Department of Electrical Engineering and Computer Science, University of Michigan. His research interests include micromachining technologies, solid-state micromachined sensors, actuators, and microelectromechanical systems; analog integrated circuits; implantable biomedical microsystems; hermetic micropackaging; and low-power wireless sensing/actuating systems. He is an Associate Editor for the Journal of Micromechanics and Microengineering of the Institute of Physics Publishing.

Dr. Najafi was awarded a National Science Foundation Young Investigator Award from 1992 to 1997, the Beatrice Winner Award for Editorial Excellence at the 1986 International Solid-State Circuits Conference, the Paul Rappaport Award for co-authoring the Best Paper published in the IEEE TRANSACTIONS ON EleCtRON DEVICES, and the Best Paper Award at ISSCC 1999. In 1994, he received the University of Michigan's "Henry Russel Award" for outstanding achievement and scholarship and was selected "Professor of the Year" in 1993. In 1998, he was named the Arthur F. Thurnau Professor for outstanding contributions to teaching and research and received the College of Engineering's Research Excellence Award. He has been active in the field of solid-state sensors and actuators for more than 18 years and has been involved in several conferences and workshops dealing with solid-state sensors and actuators, including the International Conference on Solid-State Sensors and Actuators, the Hilton Head Solid-State Sensors and Actuators Workshop, and the IEEE/ASME Micro Electromechanical Systems (MEMS) Workshop. He is the Editor of Solid-State Sensors for IEEE TRANSACTIONS ON ELECTRON DEVICES, Associate Editor for IEEE TRANSACTIONS ON BIOMEDICAL ENGINEERING, Associate Editor for IEEE JOURNAL OF SOLID-STATE CIRCUITS. 